\title{
49. Experimental Study on Autoregulation of the Cerebral Circulation
}

\author{
Keizo Kunitomi, Hatsuzo Uchida, Kosuke Ohta, Toshiki Chikamitsu, \\ Shigemasa IKedA and Futami KosAKa \\ Department of Anesthesiology, Okayama University Medical School
}

The influence of $\mathrm{PCO}_{2}$ and $\mathrm{PO}_{2}$ upon the autoregulation of the cerebral circuation was studied, after removing the neurogenic factors which control the cerebral slood flow.

Experimental method; In our experiments on 29 dogs, animals were mechanizally ventilated with the gas mixtures of nitrogen and oxygen. Bilateral lingual, sscending pharyngeal and occipital arteries were ligated, and the blood flows of the bilateral common carotid arteries and the external carotid arteries were directly masured by means of two pairs of the electromagnetic flow meters. The blood flow of the internal carotid artery was calculated from the difference of the blood flow of the common carotid artery and that of external carotid artery.

The method of denervating and blocking the nerves, which control the cerebral blood flow, is as follows; Bilateral cervical vagosympathetic nerves and sinus nerves were denervated at the same time, then bilateral superior cervical sympathetic ganglions, carotid body and aortic body were blocked by about $5 \mathrm{ml}$ of 1 per cent xylocaine. (This is called as a denervated group.) A group in which these neurogenic factors were kept intact is called as an intact group.

The following two different respiratory conditions were created by changing the concentration of the gas mixture; 1) normal $\mathrm{PCO}_{2}$ with hypoxia, 2) $\mathrm{PO}_{2}$ normal with hypercapnea.

Differences of the cerebral circulation of these two groups were compared and subjected to discussion.

Results; Blood flow in the internal carotid artery was remarkably increased by hypoxia. The rate of increase of the internal carotid blood flow was 5.6 times as great in the denervated group as in the intact group. Maximum blood flow was observed at 10 minutes after induction of hypoxia. The rate of the increase of the internal carotid blood flow was far more great compared with that of external and common carotid artery flow.

In the denervated group, the rate of the increase of internal, external and common carotid flow, blood pressure and pulse rate was not so remarkable in the hypercapneic state. But in the intact group, the increase of the internal carotid blood flow was quite remarkable. The rate of the increase was 117.9 per cent at 30 minutes after induction of hypercapnea.

Conclusion; From these experimental results described above, autoregulation of the cerebral circulation is recognized in the hypoxic state, regardless of the presence or absence of the neurogenic factors, although autoregulation is more promi- 
nent in the denervated group than in the intact group.

In the hypoxic state, we concluded that the neurogenic factors exert a suppressive action upon autoregulation of the cerebral circulation.

In the hypercapneic state, autoregulation of the cerebral circulation is observed only in the intact group. Therefore, we consider that autoregulatory mechanism of the cerebral circulation exerts through media of neurogenic factors in the hypercapneic state.

\title{
50. The Effect of Hyperventilation on Cerebral Circulation (II) Changes in CSFP, EEG, Blood Gases after Hyperventilation
}

\author{
Taira Watanabe, Hiroshi Takeshita and Akira Inamoto \\ Department of Anesthesiology, Kyoto University School of Medicine
}

In 12 subjects with normal CSFP under halothane-nitrous oxide-oxygen anesthesia, the effects of hyperventilation on CSFP, blood gases and $\mathrm{pH}$, and electroencephalogram were studied before, during and after hyperventilation.

Within 5 minutes following institution of hyperventilation, there was a rapid fall in CSFP associated with a decrease of mean arterial $\mathrm{Pco}_{2}$ from $38 \mathrm{mmHg}$ to $16 \mathrm{mmHg}$ with resultant increase of mean $\mathrm{pH}$ from 7.406 to 7.668. At the same time mean jugular $\mathrm{Po}_{2}$ decreased from $57 \mathrm{mmHg}$ to $24 \mathrm{mmHg}$.

Occurrence and duration of EEG slowing appeared within 10 minutes after the onset of hyperventilation were variable and not consistent. During entire period of hyperventilation EEG recording showed no significant change. The CSFP gradually returned and raised over the initial value at approximately 90 minutes of hyperventilation. Initial decrease in CSFP was due to well known fact of cerebral vasoconstriction produced by a decrease in arterial $\mathrm{Pco}_{2}$. Gradual return of CSFP was considered to be a redistribution between cerebral blood volume and cerebrospinal fluid volume.

As arterial $\mathrm{PCO}_{2}$ returned to near normal level after the end of hyperventilation, there was rebound phenomenon indicating rapid increase in cerebral blood flow without compensatory decrease in cerebrospinal fluid volume. EEG slowing during rebound phenomenon consisted of high voltage, 1 to $3 \mathrm{cps}$ activity. Posthyperventilation EEG slowing was reliable and consistent. The immediate cause of EEG slowing during and after hyperventilation remained obscure. Post-hyperventilation EEG slowing appeared when arterial $\mathrm{Pco}_{2}$ was normal, jugular $\mathrm{Po}_{2}$ was increased to $60 \mathrm{mmHg}$ and $\mathrm{pH}$ was significantly changed from 7.654 to 7.329 After hyperventilation arterial $\mathrm{pH}$ and base excess showed the occurrence of metabolic acidosis. Evidences observed after hyperventilation in this study sug. 